\title{
EULER SPACES OF ANALYTIC FUNCTIONS
}

\author{
JAMES ROVNYAK
}

\begin{abstract}
A formula due to Euler and Legendre is used to construct finitedifference counterparts to the Dirichlet space. The spaces have integral representations and characterizations in terms of area integrals. Their reproducing kernels are logarithms of the reproducing kernels of the Newton spaces, which are counterparts to the Hardy class. A Hilbert space with reproducing kernel

$$
\log [(1 / \bar{w} z) \log 1 /(1-\bar{w} z)]
$$

is also shown to exist and to be related to Bernoulli numbers and combinatorial theory.
\end{abstract}

1. Introduction and preliminary notions. This paper continues the plan of [5], which seeks finite-difference counterparts to notions from the theory of Hardy classes and Fourier analysis. The Hardy class for the unit disk has counterparts in the Newton spaces $\mathscr{N}_{h}, h>0$. The Euler spaces $\mathscr{E}_{h}, h>0$, defined in this paper are analogues of the Dirichlet space. Their construction is motivated by a general theorem on reproducing kernel Hilbert spaces:

Let $K(s, t)$ be the reproducing kernel for a Hilbert space $\mathscr{K}$ of functions on a set $S$. Then $H(s, t)=\exp (K(s, t))$ is the reproducing kernel for a Hilbert space $\mathscr{H}$ of functions on $S$. For any $f(t)$ in $\mathscr{K}, \exp (f(t))$ belongs to $\mathscr{H}$ and

$$
\|\exp (f(t))\|_{\mathscr{H}}^{2} \leq \exp \left(\|f(t)\|_{\mathscr{K}}^{2}\right) .
$$

If equality holds and $g(t)$ is any second element of $\mathscr{K}$, then

$$
\langle\exp (f(t)), \exp (g(t))\rangle_{\mathscr{H}}=\exp \left(\langle f(t), g(t)\rangle_{\mathscr{K}}\right) .
$$

This result is given by $\mathrm{L}$. de Branges [2] in connection with an inequality of N. A. Lebedev and I. M. Milin. Let $a_{1}, a_{2}, \ldots$ and $b_{0}, b_{1}, b_{2}, \ldots$ be complex numbers related by

$$
\exp \left(a_{1} z+a_{2} z^{2}+\cdots\right)=b_{0}+b_{1} z+b_{2} z^{2}+\cdots .
$$

Then

$$
\sum_{n=0}^{\infty}\left|b_{n}\right|^{2} \leq \exp \left(\sum_{n=1}^{\infty} n\left|a_{n}\right|^{2}\right) .
$$

A nontrivial assertion is made only when the right side is finite. The inequality follows from the theorem with $S$ equal to the unit disk in the complex plane and

$$
K(w, z)=\log \frac{1}{1-\bar{w} z}, \quad H(w, z)=\frac{1}{1-\bar{w} z} .
$$

Received by the editors March 10, 1987.

1980 Mathematics Subject Classification (1985 Revision). Primary 30H05; Secondary 44A15, $46 \mathrm{E} 20$.

Research supported by NSF Grant DMS-8701395. 
In this case $\mathscr{K}$ is the Dirichlet space of power series

$$
f(z)=a_{1} z+a_{2} z^{2}+\cdots
$$

with

$$
\|f(z)\|_{\mathscr{K}}^{2}=\sum_{n=1}^{\infty} n\left|a_{n}\right|^{2}<\infty
$$

and $\mathscr{H}$ is the space of square summable power series.

The Newton polynomials $N_{0}(z)=1$ and

$$
N_{n}(z)=(-1)^{n} \frac{z(z-1) \cdots(z-n+1)}{n !}, \quad n=1,2,3, \ldots,
$$

play the role of monomials in the finite-difference calculus:

$$
-\Delta: N_{n}(z) \rightarrow N_{n-1}(z), \quad n=0,1,2, \ldots,
$$

where $N_{-1}(z)=0$ and $\Delta: F(z) \rightarrow F(z+1)-F(z)$ is the difference operator. The Newton space $\mathcal{N}_{h}$ is the Hilbert space of holomorphic functions $F(z)$ of the form

$$
F(z)=\sum_{n=0}^{\infty} a_{n} N_{n}(z), \quad \operatorname{Re} z>-\frac{1}{2} h
$$

with

$$
\|F(z)\|_{\mathcal{N}_{h}}^{2}=\left|a_{0}\right|^{2}+\frac{h}{1 !}\left|a_{1}\right|^{2}+\frac{h(h+1)}{2 !}\left|a_{2}\right|^{2}+\cdots<\infty .
$$

The weighted Mellin transform of a function $f(t)$ in $L^{2}\left(w_{h}\right)$, where $w_{h}(t)=e^{-t} t^{h-1}$ on $(0, \infty)$, is the function

$$
F(z)=\frac{1}{\Gamma(z+h)} \int_{0}^{\infty} f(t) e^{-t} t^{z+h-1} d t, \quad \operatorname{Re} z>-\frac{1}{2} h .
$$

The mapping $f(t) \rightarrow F(z)$ so defined is an isometry from $L^{2}\left(w_{h}\right)$ onto $\mathscr{N}_{h}$. Details and additional information may be found in [5].

For any positive number $h$, the Newton space $\mathscr{N}_{h}$ has reproducing kernel

$$
\frac{\Gamma(h) \Gamma(z+\bar{w}+h)}{\Gamma(z+h) \Gamma(\bar{w}+h)}, \quad \operatorname{Re} z>-\frac{1}{2} h, \operatorname{Re} w>-\frac{1}{2} h .
$$

The logarithmic version of $\mathscr{N}_{h}$, if there is one, should have reproducing kernel

$$
\log \frac{\Gamma(h) \Gamma(z+\bar{w}+h)}{\Gamma(z+h) \Gamma(\bar{w}+h)}, \quad \operatorname{Re} z>-\frac{1}{2} h, \operatorname{Re} w>-\frac{1}{2} h .
$$

We show that a space $\mathscr{E}_{h}$ with this reproducing kernel does indeed exist, and its structure is determined by an identity that is due to Euler in the case $h=1$ and Legendre in the general case. An Euler transform is defined analogously to the weighted Mellin transform, and it yields an isometric integral representation of the space. Moreover, the space is characterized by certain area integrals of derivatives in a manner reminiscent of a well-known characterization of the Dirichlet space.

It is natural to ask if additional logarithms can be formed. In this direction, we show that the kernel

$$
\log \left[\frac{1}{\bar{w} z} \log \frac{1}{1-\bar{w} z}\right]
$$

is positive definite on the unit disk and related to Bernoulli numbers and combinatorial theory. It is not known if a similar result holds for the Euler spaces. 
2. Euler spaces. We first derive the Euler-Legendre formula [6, §73]

$$
\log \frac{\Gamma(h) \Gamma(z+\bar{w}+h)}{\Gamma(z+h) \Gamma(\bar{w}+h)}=\int_{0}^{1} \frac{t^{z}-1}{t-1} \frac{t^{\bar{w}}-1}{t-1} \frac{t-1}{\log t} t^{h-1} d t,
$$

which structures everything that follows. The principal branch of logarithm is understood $(\log 1=0)$. The formula is valid with absolute convergence of the integral for any complex numbers $z, w, h$ such that $h, z+\bar{w}+h, z+h, \bar{w}+h$ have positive real parts. In particular, the formula holds when $h$ is real and positive, $\operatorname{Re} z>-\frac{1}{2} h$, and $\operatorname{Re} w>-\frac{1}{2} h$. Begin with Gauss's formula [6, §72]

$$
\frac{d}{d z} \log \Gamma(z)=\int_{0}^{\infty}\left[\frac{e^{-t}}{t}-\frac{e^{-t z}}{1-e^{-t}}\right] d t, \quad \operatorname{Re} z>0,
$$

in its integrated form due to Plana [6, §73],

$$
\log \Gamma(z)=\int_{0}^{\infty}\left[z-1-\frac{1-e^{-(z-1) s}}{1-e^{-s}}\right] \frac{e^{-s}}{s} d s, \quad \operatorname{Re} z>0 .
$$

If $h, z+\bar{w}+h, z+h, \bar{w}+h$ have positive real parts, then

$$
\begin{aligned}
\log \frac{\Gamma(h) \Gamma(z+\bar{w}+h)}{\Gamma(z+h) \Gamma(\bar{w}+h)}= & \int_{0}^{\infty}\left[h-1-\frac{1-e^{-(h-1) s}}{1-e^{-s}}\right] \frac{e^{-s}}{s} d s \\
& +\int_{0}^{\infty}\left[z+\bar{w}+h-1-\frac{1-e^{-(z+\bar{w}+h-1) s}}{1-e^{-s}}\right] \frac{e^{-s}}{s} d s \\
& -\int_{0}^{\infty}\left[z+h-1-\frac{1-e^{-(z+h-1) s}}{1-e^{-s}}\right] \frac{e^{-s}}{s} d s \\
& -\int_{0}^{\infty}\left[\bar{w}+h-1-\frac{1-e^{-(\bar{w}+h-1) s}}{1-e^{-s}}\right] \frac{e^{-s}}{s} d s \\
= & \int_{0}^{\infty}\left(e^{-z s}-1\right)\left(e^{-\bar{w} s}-1\right) \frac{e^{-h s}}{s\left(1-e^{-s}\right)} d s
\end{aligned}
$$

which yields (2) after a change of variables.

THEOREM 1. For any positive number $h,(2)$ is the reproducing kernel for a Hilbert space $\mathscr{E}_{h}$ of holomorphic functions on the half-plane $\operatorname{Re} z>-\frac{1}{2} h$.

The spaces $\mathscr{E}_{h}, h>0$, are called Euler spaces.

ProOF. By (2),

$$
\begin{aligned}
& \sum_{j, k=1}^{n} c_{j} \bar{c}_{k} \log \frac{\Gamma(h) \Gamma\left(w_{k}+\bar{w}_{j}+h\right)}{\Gamma\left(w_{k}+h\right) \Gamma\left(\bar{w}_{j}+h\right)} \\
& \quad=\int_{0}^{1}\left|\sum_{j=1}^{n} c_{j} \frac{t^{w_{j}}-1}{t-1}\right|^{2} \frac{t-1}{\log t} t^{h-1} d t \geq 0
\end{aligned}
$$

for any numbers $w_{1}, \ldots, w_{n}$ in the half-plane $\operatorname{Re} w>-\frac{1}{2} h$ and all complex numbers $c_{1}, \ldots, c_{n}$. The existence of the space thus follows from a well-known construction in the theory of reproducing kernel Hilbert spaces [1]. 
For any positive number $h$, let $L^{2}\left(\lambda_{h}\right)$ be the Lebesgue space of measurable functions on $(0,1)$ square summable with respect to

$$
\lambda_{h}(t)=\frac{t-1}{\log t} t^{h-1} .
$$

The weight function is positive and integrable on $(0,1)$, with

$$
\int_{0}^{1} \lambda_{h}(t) d t=\log \frac{\Gamma(h) \Gamma(h+2)}{\Gamma(h+1) \Gamma(h+1)}=\log (1+1 / h)
$$

by the Euler-Legendre formula (2). Define the Euler transform of any element $f(t)$ of $L^{2}\left(\lambda_{h}\right)$ to be the function

$$
F(z)=\int_{0}^{1} f(t) \frac{t^{z}-1}{t-1} \lambda_{h}(t) d t, \quad \operatorname{Re} z>-\frac{1}{2} h .
$$

Any such function vanishes at the origin. The Euler transform is an integrated form of the Mellin transform.

THEOREM 2. The Euler transform of a function $f(t)$ in $L^{2}\left(\lambda_{h}\right)$ is a holomorphic function $F(z)$ on the half-plane $\operatorname{Re} z>-\frac{1}{2} h$. If $F(z) \equiv 0$ on the half-plane, then $f(t)=0$ a.e. on $(0,1)$. Differentiation under the integral is justified in the Euler transform and yields

$$
F^{\prime}(z)=\int_{0}^{1} t^{z+h-1} f(t) d t
$$

for $\operatorname{Re} z>-\frac{1}{2} h$.

PROOF. The identity

$$
\frac{t^{z}-t^{w}}{z-w}=\log t \int_{0}^{1} t^{s z+(1-s) w} d s
$$

implies the estimate

$$
\left|\frac{t^{z}-t^{w}}{z-w}\right|<t^{-\delta} \log \frac{1}{t}, \quad 0<t<1, \operatorname{Re} z \geq-\delta, \operatorname{Re} w \geq-\delta
$$

for any number $\delta<\frac{1}{2} h$. Then

$$
\int_{0}^{1}\left|\frac{t^{z}-1}{t-1}\right|^{2} \lambda_{h}(t) d t \leq C_{\delta}|z|^{2}, \quad \operatorname{Re} z \geq-\delta
$$

for some $C_{\delta}>0$. In particular, the integral defining $F(z)$ is absolutely convergent for $\operatorname{Re} z>-\frac{1}{2} h$. Differentiation under the integral in (3) is justified, since

$$
\left|\frac{t^{z}-t^{w}}{z-w} \frac{1}{t-1} f(t) \lambda_{h}(t)\right| \leq t^{-\delta} \frac{\log t}{t-1}|f(t)| \lambda_{h}(t),
$$

$\operatorname{Re} z \geq-\delta, \operatorname{Re} w \geq-\delta\left(\delta<\frac{1}{2} h\right)$. Hence (4) follows. If $F(z)$ vanishes identically, so does $F^{\prime}(z)$. In this case $f(t)=0$ a.e. on $(0,1)$ by the uniqueness theorem for Mellin transforms. 
THEOREM 3. For any positive number $h$, the Euler transform (3) defines an isometry $f(t) \rightarrow F(z)$ from $L^{2}\left(\lambda_{h}\right)$ onto $\mathscr{E}_{h}$.

PrOOF. By Theorem 2, there is a unique Hilbert space $\mathscr{K}$ of functions on $\operatorname{Re} z>$ $-\frac{1}{2} h$ such that the mapping $f(t) \rightarrow F(z)$ defined by the Euler transform is an isometry from $L^{2}\left(\lambda_{h}\right)$ onto $\mathscr{K}$. By the definition of the space, $\mathscr{K}$ has reproducing kernel

$$
\log \frac{\Gamma(h) \Gamma(z+\bar{w}+h)}{\Gamma(z+h) \Gamma(\bar{w}+h)}=\int_{0}^{1} \frac{t^{z}-1}{t-1} \frac{t^{\bar{w}}-1}{t-1} \lambda_{h}(t) d t,
$$

$\operatorname{Re} z>-\frac{1}{2} h, \operatorname{Re} w>-\frac{1}{2} h$. Since $\mathscr{K}$ and $\mathscr{E}_{h}$ have the same reproducing kernel, they are equal isometrically.

A function $\varphi(\xi)$ on a real interval $(a, \infty)$ is completely monotonic if for any $\xi$ in the interval and any number $\delta>0$,

$$
\sum_{j=0}^{n}(-1)^{j}\left(\begin{array}{l}
n \\
j
\end{array}\right) \varphi(\xi+j \delta) \geq 0
$$

for every $n=0,1,2, \ldots$. The condition asserts that all finite differences of the function are nonnegative. The notion arises in the theory of the Hausdorff moment problem [9, pp. 86-89].

THEOREM 4. Let $h$ be any positive number, and let $F(z)$ be a holomorphic function on the half-plane $\operatorname{Re} z>-\frac{1}{2} h$ such that $F(0)=0$. Then $F(z)$ belongs to $\mathscr{E}_{h}$ if and only if

$$
\varphi_{F}(\xi)=\frac{1}{\pi} \iint_{\xi<x<\xi+1 / 2}\left|F^{\prime}(x+i y)\right|^{2} d x d y
$$

is a bounded and completely monotonic function of $\xi>-\frac{1}{2} h$. In this case,

$$
\|F\|_{\varepsilon_{h}}^{2}=\sup _{\xi>-h / 2} \varphi_{F}(\xi)=\frac{1}{\pi} \iint_{-h / 2<x<-h / 2+1 / 2}\left|F^{\prime}(x+i y)\right|^{2} d x d y .
$$

PROOF. The Mellin reciprocal relations

$$
\begin{aligned}
& v(x)=(2 \pi)^{-1 / 2} \int_{0}^{\infty} u(t) t^{-1 / 2+i x} d t, \\
& u(x)=(2 \pi)^{-1 / 2} \int_{-\infty}^{\infty} v(t) x^{-1 / 2-i t} d t,
\end{aligned}
$$

determine an isometric correspondence between functions $u(x)$ in $L^{2}(0, \infty)$ and functions $v(x)$ in $L^{2}(-\infty, \infty)$. Both integrals are taken in the mean square sense.

Suppose first that $F(z)$ belongs to $\mathscr{E}_{h}$. Let $F(z)$ be the Euler transform (3) of the element $f(t)$ of $L^{2}\left(\lambda_{h}\right)$. By the Mellin representation (4) of $F^{\prime}(z)$,

$$
\int_{-\infty}^{\infty}\left|F^{\prime}(x+i y)\right|^{2} d y=2 \pi \int_{0}^{1} t^{2 x+2 h-1}|f(t)|^{2} d t
$$


for any $x>-\frac{1}{2} h$. Therefore for any $\xi>-\frac{1}{2} h$,

$$
\begin{aligned}
\varphi_{F}(\xi) & =\frac{1}{\pi} \iint_{\xi<x<\xi+1 / 2}\left|F^{\prime}(x+i y)\right|^{2} d x d y \\
& =2 \int_{0}^{1} \int_{\xi}^{\xi+1 / 2} t^{2 x+2 h-1} d x|f(t)|^{2} d t \\
& =\int_{0}^{1} t^{2 \xi+h}|f(t)|^{2} \lambda_{h}(t) d t .
\end{aligned}
$$

It follows that $\varphi_{F}(\xi)$ is a bounded function of $\xi>-\frac{1}{2} h$, and

$$
\begin{aligned}
\|F\|_{\mathscr{E}_{h}}^{2} & =\int_{0}^{1}|f(t)|^{2} \lambda_{h}(t) d t=\lim _{\xi \downarrow-h / 2} \varphi_{F}(\xi) \\
& =\frac{1}{\pi} \iint_{-h / 2<x<-h / 2+1 / 2}\left|F^{\prime}(x+i y)\right|^{2} d x d y .
\end{aligned}
$$

The function $\varphi_{F}(\xi)$ is completely monotonic for $\xi>-\frac{1}{2} h$, since

$$
\begin{aligned}
\sum_{j=0}^{n}( & -1)^{j}\left(\begin{array}{l}
n \\
j
\end{array}\right) \varphi_{F}(\xi+j \delta) \\
& =\int_{0}^{1} \sum_{j=0}^{n}(-1)^{j}\left(\begin{array}{l}
n \\
j
\end{array}\right) t^{2 \xi+2 j \delta+h}|f(t)|^{2} \lambda_{h}(t) d t \\
& =\int_{0}^{1}\left(1-t^{2 \delta}\right)^{n} t^{2 \xi+h}|f(t)|^{2} \lambda_{h}(t) d t \\
& \geq 0
\end{aligned}
$$

for all $\xi>-\frac{1}{2} h, \delta>0$, and $n=0,1,2, \ldots$.

Conversely, suppose that $\varphi_{F}(\xi)$ is bounded and completely monotonic for $\xi>$ $-\frac{1}{2} h$. Standard arguments from Fourier analysis show that $F^{\prime}(z)$ belongs to the Hardy class $H^{2}(a, b)$ for any strip $a<x<b,-\frac{1}{2} h<a<b<\infty$. A theorem of Paley and Wiener [8] gives the representation

$$
F^{\prime}(z)=\int_{-\infty}^{\infty} e^{s z \mathscr{F}}(s) d s=\int_{0}^{\infty} t^{z+h-1} f(t) d t,
$$

$\operatorname{Re} z>-\frac{1}{2} h$, where $\mathscr{F}(s)$ and $f(t)=t^{-h} \mathscr{F}(\log t)$ are measurable functions such that

$$
\int_{-\infty}^{\infty} e^{2 x s}|\mathscr{F}(s)|^{2} d s=\int_{0}^{\infty} t^{2 x+2 h-1}|f(t)|^{2} d t<\infty
$$

for all $x>-\frac{1}{2} h$. Therefore for any $x>-\frac{1}{2} h$,

$$
\int_{-\infty}^{\infty}\left|F^{\prime}(x+i y)\right|^{2} d y=2 \pi \int_{0}^{\infty} t^{2 x+2 h-1}|f(t)|^{2} d t
$$

and for any $\xi>-\frac{1}{2} h$,

$$
\frac{1}{\pi} \iint_{\xi<x<\xi+1 / 2}\left|F^{\prime}(x+i y)\right|^{2} d x d y=\int_{0}^{\infty} t^{2 \xi+h}|f(t)|^{2} \lambda_{h}(t) d t .
$$


By hypothesis, the left side of the last identity is a bounded and completely monotonic function of $\xi>-\frac{1}{2} h$. A calculation similar to one used above shows that

$$
\int_{0}^{\infty}\left(1-t^{2 \delta}\right)^{n} t^{2 \xi+h}|f(t)|^{2} \lambda_{h}(t) d t \geq 0
$$

for all $\xi>-\frac{1}{2} h, \delta>0$, and $n=0,1,2, \ldots$ For $n$ odd the inequality implies

$$
\begin{gathered}
\int_{1}^{\infty}\left|1-t^{2 \delta}\right|^{n} t^{2 \xi+h}|f(t)|^{2} \lambda_{h}(t) d t \\
\leq \int_{0}^{1} t^{2 \xi+h}|f(t)|^{2} \lambda_{h}(t) d t .
\end{gathered}
$$

It follows that $f(t)=0$ a.e. for $t>1$. By Theorem 2 ,

$$
G(z)=\int_{0}^{1} f(t) \frac{t^{z}-1}{t-1} \lambda_{h}(t) d t, \quad \operatorname{Re} z>-\frac{1}{2} h
$$

defines a function in $\mathscr{E}_{h}$. Since

$$
F^{\prime}(z)=\int_{0}^{1} t^{z+h-1} f(t) d t=G^{\prime}(z)
$$

identically for $\operatorname{Re} z>-\frac{1}{2} h$ and $F(0)=G(0)=0, F(z)$ and $G(z)$ are equal identically. Therefore $F(z)$ belongs to $\mathscr{E}_{h}$.

EXAMPLE 1. Fix $h>0$, and let $\alpha_{1}, \ldots, \alpha_{r}$ be complex numbers with positive real parts. For any numbers $c_{1}, \ldots, c_{r}$, consider

$$
F(z)=\prod_{j=1}^{r}\left(1+z /\left(\alpha_{j}+\frac{1}{2} h\right)\right)^{c_{j}}, \quad \operatorname{Re} z>-\frac{1}{2} h .
$$

Then $\log F(z)$ belongs to $\mathscr{E}_{h}$, and

$$
\|\log F(z)\|_{\mathscr{E}_{h}}^{2}=\sum_{j, k=1}^{r} c_{j} \bar{c}_{k} \log \left(1+1 /\left(\alpha_{j}+\bar{\alpha}_{k}\right)\right) .
$$

The function $F(z)$ belongs to the Newton space $\mathscr{N}_{h}$, and

$$
\|F(z)\|_{\mathscr{N}_{h}}^{2} \leq \prod_{j=1}^{r}\left(1+1 /\left(\alpha_{j}+\bar{\alpha}_{k}\right)\right)^{c_{j} \bar{c}_{k}}
$$

The example includes every rational function $F(z)$ that has no zeros or poles in the closed half-plane $\operatorname{Re} z \geq-\frac{1}{2} h$. The Newton-space estimate is a special case of the finite-difference counterpart of the Lebedev-Milin inequality stated in $§ 1$.

To obtain the example, write the Euler-Legendre formula (2) with $w=1$ and $h$ replaced by $h+\frac{1}{2} \alpha$ for any number $\alpha$ with positive real part. This gives

$$
\begin{aligned}
\int_{0}^{1} \frac{t^{z}-1}{t-1} t^{\alpha-h / 2} \frac{t-1}{\log t} t^{h-1} d t & =\log \frac{\Gamma\left(\alpha+\frac{1}{2} h\right) \Gamma\left(z+1+\alpha+\frac{1}{2} h\right)}{\Gamma\left(z+\alpha+\frac{1}{2} h\right) \Gamma\left(1+\alpha+\frac{1}{2} h\right)} \\
& =\log \left(1+z /\left(\alpha+\frac{1}{2} h\right)\right)
\end{aligned}
$$


for $\operatorname{Re} z>-\frac{1}{2} h$. It follows that $\log F(z)$ is the Euler transform of the function

$$
f(t)=\sum_{j=1}^{r} c_{j} t^{\alpha_{j}-h / 2}
$$

in $L^{2}\left(\lambda_{h}\right)$. The formula for the square of the norm of $\log F(z)$ in the Euler space $\mathscr{E}_{h}$ follows from a straightforward calculation of the square of the norm of $f(t)$ in $L^{2}\left(\lambda_{h}\right)$; this uses the Euler-Legendre formula (2) one more time. The estimate of the square of the norm of $F(z)$ in the Newton space then follows from the theorem on reproducing kernel Hilbert spaces stated in $\S 1$.

EXAMPLE 2. Let $h$ be any positive number. Every $F(z)$ in $\mathscr{C}_{h}$ has a unique representation as a Newton series

$$
F(z)=\sum_{n=1}^{\infty} a_{n} N_{n}(z), \quad \operatorname{Re} z>-\frac{1}{2} h,
$$

with coefficients given by

$$
a_{n}=-\int_{0}^{1}(1-t)^{n-1} \lambda_{h}(t) f(t) d t, \quad n=1,2,3, \ldots,
$$

where $f(t)$ is the element of $L^{2}\left(\lambda_{h}\right)$ corresponding to $F(z)$ by means of the Euler transform (3).

A formal calculation using the binomial series yields

$$
\begin{aligned}
F(z) & =\int_{0}^{1} \frac{t^{z}-1}{t-1} \lambda_{h}(t) f(t) d t \\
& =-\int_{0}^{1} \frac{1}{1-t}\left[(1-(1-t))^{z}-1\right] \lambda_{h}(t) f(t) d t \\
& =\sum_{n=1}^{\infty} a_{n} N_{n}(z)
\end{aligned}
$$

with coefficients given by (6). To justify the calculation, it is sufficient to show that

$$
\sum_{n=1}^{\infty}\left|N_{n}(z)\right| \int_{0}^{1}\left|(1-t)^{n-1} \lambda_{h}(t) f(t)\right| d t<\infty
$$

for $\operatorname{Re} z>-\frac{1}{2} h$.

We use standard information concerning the behavior of the gamma function for large arguments $[3, \S 1.18]$. Let $a$ and $b$ be any complex numbers, and let $\delta$ be a positive number less than $\pi$. Then there are positive constants $M$ and $K$ such that

$$
\frac{\Gamma(z+a)}{\Gamma(z+b)}=z^{a-b}[1+\varepsilon(z)]
$$

with $|\varepsilon(z)| \leq K /|z|$ for $|\arg z|<\pi-\delta,|z|>M$. In particular, for any complex number $z$ there is a positive constant $C_{z}$ such that

$$
\left|N_{n}(z)\right|=\left|\frac{\Gamma(n-z)}{\Gamma(n+1) \Gamma(-z)}\right| \leq C_{z} n^{-z-1}
$$


for every positive integer $n$. By Schwarz's inequality,

$$
\begin{aligned}
\int_{0}^{1}\left|(1-t)^{n-1} \lambda_{h}(t) f(t)\right| d t & \leq \text { Const } \cdot\left\{\int_{0}^{1}(1-t)^{2 n-2} \lambda_{h}(t) d t\right\}^{1 / 2} \\
& \leq \text { Const } \cdot\left\{\int_{0}^{1}(1-t)^{2 n-2} t^{h-1} d t\right\}^{1 / 2} \\
& =\text { Const } \cdot\left[\frac{\Gamma(2 n-1) \Gamma(h)}{\Gamma(2 n+h-1)}\right]^{1 / 2} \\
& \leq \text { Const } \cdot n^{-\frac{1}{2} h}
\end{aligned}
$$

for every positive integer $n$. It follows that (5) holds with the particular choice of coefficients (6).

The representation (5) is unique because it holds throughout the half-plane $\operatorname{Re} z>-\frac{1}{2} h$ [7, p. 225]. Due to the phenomenon of null series [7, pp. 224-225], there are other Newton series which represent $F(z)$ in smaller half-planes, such as $\operatorname{Re} z>0$.

EXAMPLE 3 . The only polynomial in $\mathscr{E}_{h}, h$ any positive number, is the polynomial identically zero. More generally, a Newton series with gaps represents a function in $\mathscr{E}_{h}$ only if it vanishes identically.

To be precise, let the Newton series

$$
F(z)=\sum_{n=1}^{\infty} a_{n} N_{n}(z)
$$

converge for $\operatorname{Re} z>-\frac{1}{2} h$ and represent a function in $\mathscr{E}_{h}$, and suppose $\sum_{a_{n} \neq 0} 1 / n$ $<\infty$. If $f(t)$ is the corresponding element of $L^{2}\left(\lambda_{h}\right)$, then by the preceding example,

$$
\int_{0}^{1}(1-t)^{n-1} \lambda_{h}(t) f(t) d t=0
$$

for a set $S$ of positive integers $n$ such that $\sum_{n \in S} 1 / n=\infty$. By the Müntz-Szász theorem, $f(t)=0$ a.e., and so $F(z)$ vanishes identically.

3. Iteration of the logarithm. The expression

$$
\log \left[\log \frac{\Gamma(h) \Gamma(z+\bar{w}+h)}{\Gamma(z+h) \Gamma(\bar{w}+h)}\right]
$$

is not a candidate for a new reproducing kernel: the expression reduces to $\log 0$ for $z=w=0$. One can ask, however, if

$$
\log \left[\frac{1}{z \bar{w}} \log \frac{\Gamma(h) \Gamma(z+\bar{w}+h)}{\Gamma(z+h) \Gamma(\bar{w}+h)}\right]
$$

is the reproducing kernel for some Hilbert space of analytic functions on the halfplane $\operatorname{Re} z>-\frac{1}{2} h$. We do not know if this is the case, but some support for an affirmative answer is given by an analogous result for the Dirichlet space on the unit disk. The new space has a surprising connection with combinatorial theory. 
THEOREM 5. The function

$$
\log \left[\frac{1}{z \bar{w}} \log \frac{1}{1-z \bar{w}}\right], \quad|w|<1,|z|<1,
$$

is the reproducing kernel for some Hilbert space of holomorphic functions on the unit disk.

The Bernoulli numbers of the second kind are the numbers $b_{0}, b_{1}, b_{2}, \ldots$ in the expansion [4, p. $279(9)]$

$$
\frac{\varsigma}{\log (1+\varsigma)}=\sum_{n=0}^{\infty} b_{n} \varsigma^{n} \text {. }
$$

A recursive computation yields $b_{0}=1$ and

$$
\begin{aligned}
b_{1} & =\frac{1}{2}, & b_{2} & =-\frac{1}{12}, \\
b_{3} & =\frac{1}{24}, & b_{4} & =-\frac{19}{720}, \\
b_{5} & =\frac{3}{160}, & b_{6} & =-\frac{363}{60480}, \\
b_{7} & =\frac{275}{24192}, & b_{8} & =-\frac{33953}{3528800}, \\
b_{9} & =\frac{8183}{1036800}, & b_{10} & =-\frac{3250433}{479001600}, \\
b_{11} & =\frac{4671}{788480}, & b_{12} & =-\frac{13695779093}{2615348736000} .
\end{aligned}
$$

PROOF OF THEOREM 5. It is sufficient to show that the coefficients in the expansion

$$
\log \left[\frac{1}{\varsigma} \log \frac{1}{1-\varsigma}\right]=c_{1} \varsigma+c_{2} \varsigma^{2}+c_{3} \varsigma^{3}+\cdots
$$

are positive. To this end, write

$$
\frac{1}{\varsigma} \log \frac{1}{1-\varsigma}=\frac{1}{1-w}
$$

Then

$$
w=1-\frac{-\varsigma}{\log (1-\varsigma)}=b_{1} \varsigma-b_{2} \varsigma^{2}+b_{3} \varsigma^{3}-\cdots
$$

and so

$$
\begin{aligned}
\log \left[\frac{1}{\zeta} \log \frac{1}{1-\varsigma}\right]= & \log \frac{1}{1-w} \\
= & w+\frac{1}{2} w^{2}+\frac{1}{3} w^{3}+\cdots \\
= & \left(b_{1} \varsigma-b_{2} \varsigma^{2}+b_{3} \varsigma^{3}-\cdots\right) \\
& +\frac{1}{2}\left(b_{1} \varsigma-b_{2} \varsigma^{2}+b_{3} \varsigma^{3}-\cdots\right)^{2} \\
& +\frac{1}{3}\left(b_{1} \varsigma-b_{2} \varsigma^{2}+b_{3} \varsigma^{3}-\cdots\right)^{3} \\
& +\cdots .
\end{aligned}
$$


All coefficients are positive since $b_{2 n-1}>0$ and $b_{2 n}<0$ for every positive integer $n[4$, p. 267].

The preceding argument yields a formula for the coefficients $c_{1}, c_{2}, c_{3}, \ldots$ in (7). While the formula shows the positivity of coefficients, it is otherwise not illuminating. To derive a better representation of the numbers, write

$$
\begin{aligned}
1+\sum_{1}^{\infty} j c_{j} \varsigma^{j}= & 1+\varsigma \frac{d}{d \zeta} \log \left[\frac{1}{\zeta} \log \frac{1}{1-\varsigma}\right] \\
= & \frac{1}{1-\varsigma} \frac{\varsigma}{\log (1 /(1-\varsigma))} \\
= & \left(1+\varsigma+\varsigma^{2}+\cdots\right)\left(b_{0}-b_{1} \varsigma+b_{2} \varsigma^{2}-\cdots\right) \\
= & b_{0}+\left(b_{0}-b_{1}\right) \varsigma+\left(b_{0}-b_{1}+b_{2}\right) \varsigma^{2}+\cdots \\
& +\left(b_{0}-b_{1}+b_{2}-\cdots+(-1)^{n} b_{n}\right) \varsigma^{n}+\cdots \\
&
\end{aligned}
$$

Thus

$$
c_{n}=\frac{1}{n}\left[b_{0}-b_{1}+b_{2}-\cdots+(-1)^{n} b_{n}\right]
$$

for every $n=1,2,3, \ldots$. This yields

$$
\begin{aligned}
c_{1} & =\frac{1}{2}, & c_{7} & =\frac{751}{17280}, \\
c_{2} & =\frac{5}{24}, & c_{8} & =\frac{1070017}{29030400}, \\
c_{3} & =\frac{1}{8}, & c_{9} & =\frac{2857}{89600}, \\
c_{4} & =\frac{251}{28800}, & c_{10} & =\frac{26843353}{958003200}, \\
c_{5} & =\frac{19}{288}, & c_{11} & =\frac{434293}{17418240}, \\
c_{6} & =\frac{19087}{362880}, & c_{12} & =\frac{703604254357}{31384184832000} .
\end{aligned}
$$

The representation (8) does not immediately imply the positivity of the numbers $c_{1}, c_{2}, c_{3}, \ldots$ An alternative proof of positivity follows on combining (8) with the known identities [4, p. 268 (8) and p. 265 (2)], which yields

$$
c_{n}=\frac{1}{n} \int_{0}^{1} N_{n}(t-1) d t>0
$$

for every positive integer $n$. \{Caution: The formula for $\psi_{n}(-1)$ on line 4 of p. 267 of [4] should not be used because it contains a misprint in the upper limit of the summation index.\}

\section{REFERENCES}

1. N. Aronszajn, Theory of reproducing kernels, Trans. Amer. Math. Soc. 68 (1950), 337-404.

2. L. de Branges, Square summable power series, Springer-Verlag (in preparation).

3. A. Erdélyi, et al., Higher transcendental functions, vol. 1, McGraw-Hill, New York, 1953.

4. C. Jordan, Calculus of finite differences, 3rd ed., Chelsea, New York, 1979. 
5. C. Markett, M. Rosenblum, and J. Rovnyak, A Plancherel theory for Newton spaces, Integral Equations and Operator Theory 9 (1986), 831-862.

6. N. Nielsen, Handbuch der Theorie der Gammafunktion, Chelsea, New York, 1965.

7. N. E. Nörlund, Vorlesungen über Differenzenrechnung, Chelsea, New York, 1954.

8. R. E. A. C. Paley and N. Wiener, Fourier transforms in the complex domain, Amer. Math. Soc. Colloq. Publ., vol. 19, Amer. Math. Soc., Providence, R.I., 1934.

9. J. A. Shohat and J. D. Tamarkin, The problem of moments, Math. Surveys, no. 1, Amer. Math. Soc., Providence, R.I., 1943.

Department of Mathematics, Mathematics-Astronomy Building, UniverSity OF Virginia, ChaRlotTESVILle, Virginia 22903 